\title{
1D Frustrated Ferromagnetic Model with Added Dzyaloshinskii-Moriya Interaction
}

\author{
Javad Vahedi ${ }^{1}$, Saeed Mahdavifar ${ }^{2}$ \\ ${ }^{1}$ Physics Research Center, Science Research Branch, \\ Islamic Azad University, 19585-466, Tehran, Iran and \\ 2 Department of Physics, University of Guilan, 41335-1914,Rasht, Iran
}

(Dated: October 16, 2018)

\begin{abstract}
The one-dimensional (1D) isotropic frustrated ferromagnetic spin-1/2 model is considered. Classical and quantum effects of adding a Dzyaloshinskii-Moriya (DM) interaction on the ground state of the system is studied using the analytical cluster method and numerical Lanczos technique. Cluster method results, show that the classical ground state magnetic phase diagram consists of only one single phase: "chiral". The quantum corrections are determined by means of the Lanczos method and a rich quantum phase diagram including the gapless Luttinger liquid, the gapped chiral and dimer orders is obtained. Moreover, next nearest neighbors will be entangled by increasing DM interaction and for open chains, end-spins are entangled which shows the long distance entanglement (LDE) feature that can be controlled by DM interaction.

PACS numbers: 75.10.Jm; 75.10.Pq
\end{abstract}

\section{INTRODUCTION}

An important goal in study of quantum spin systems is the search for novel phases emerging from competing interactions between particles. Low dimensional quantum spin systems typically exhibit strongly correlated effects which drive them toward new regimes with no classical analog. Many properties of the systems in these regimes or "quantum phases" can be understood if we explore their ground state and low-lying excitations.

Therefore a significant fraction of current research on such systems has focussed on understanding of frustrated systems, which exhibits a variety of exotic quantum states ${ }^{1}$. In particular, the frustrated spin chains are described by competing interactions between the nearestneighbor $\left(J_{1}\right)$ and next-nearest-neighbor $\left(J_{2}\right)$ interactions. The Hamiltonian of the model is given by

$$
H=\sum_{j=1}^{N}\left[J_{1} \vec{S}_{j} \cdot \vec{S}_{j+1}+J_{2} \vec{S}_{j} \cdot \vec{S}_{j+2}\right],
$$

where $\mathbf{S}_{j}$ represents the $S=1 / 2$ operator at $j$-th site of the chain. The model with both antiferromagnetic interactions $J_{1}, J_{2}>0$ is well studied ${ }^{2-10}$. Also the model (11) with ferromagnetic and antiferromagnetic interactions $\left(J_{1}<0, J_{2}>0\right)$ (frustrated ferromagnetic model) has been a subject of many studies $11-14$. It is known that the ground state is ferromagnetic for $\alpha=\frac{J_{2}}{\left|J_{1}\right|}<\frac{1}{4}$. At $\alpha_{c}=\frac{1}{4}$ the ferromagnetic state is degenerate with a singlet state. The wave function of this singlet state is exactly known $\frac{15.16}{}$. For $\alpha>\frac{1}{4}$, the ground state is an incommensurate singlet. It has been long believed that at $\alpha>\frac{1}{4}$ the model is gapless $\frac{7.18}{}$ but the one-loop renormalization group analysis indicates $\frac{13,19}{19}$ that the gap opens to a Lorentz symmetry breaking perturbation. However, existence of the energy gap has not been yet verified numerically $\underline{13}$. Using field theory considerations it has been proposed 17 that a very tiny but finite gap exists which can be hardly observed by numerical techniques.

In vicinity of $\alpha=\frac{1}{4}$, the singlet ground state energy behaves as $E_{0}=\left(\alpha-\frac{1}{4}\right)^{\beta}$, where $\beta$ is a critical exponent. Using variational approaches $\frac{20}{}$, it has been shown that the quantum fluctuations definitely change the classical critical exponent $\underline{\underline{7} .14}$ and yields $\beta=\frac{5}{3}$ which has been confirmed numerically $\stackrel{21}{2}$.

Beside a general interest in understanding frustrations and phase transitions in the model systems described by the Hamiltonian in $\mathrm{Eq}(1)$, it helps people to understand intriguing magnetic properties of a novel class of edge-sharing copper oxides, described by the F-AF frustrated model ${ }^{22-24}$. Recently some novel magnetic properties were discovered in a variety of quasi-one dimensional materials that are known to belong to the class of Dzyaloshinskii-Moriya (DM) $\left(\vec{D} \cdot\left(\vec{S}_{i} \times \vec{S}_{j}\right)\right)$ magnet to explain helical magnetic structures. The relevance of antisymmetric superexchange interactions in spin Hamiltonians which describe quantum antiferromagnetic systems was introduced phenomenologically by Dzyaloshinskii 25 . Moriya showed later, that such interactions arise naturally in the perturbation theory due to the spin-orbit coupling in magnetic systems with low symmetry ${ }^{26}$.

Some multiferroics cuprates, such as $\mathrm{LiCuVO}_{4}{ }^{27,28}$, $\mathrm{LiCu}_{2} \mathrm{O}_{2} \stackrel{29}{ }$ and $\mathrm{Cu}_{2} \mathrm{GeO}_{4}$, are expected to be described by DM interaction. This has stimulated extensive investigations of various properties which are created by the DM interaction. However, it is difficult to handle the DM interaction analytically and interpret experimental data. A Numerical analysis then helps us to understand experimental observation and even expand our knowledge about many interesting quantum phenomena of lowdimensional quantum magnets. In the present work, we address a different problem in the subject of the frustrated ferromagnetic spin- $1 / 2$ chains. We consider the $1 \mathrm{D}$ spin- $1 / 2$ frustrated ferromagnetic model with added DM interaction and study the classical and quantum magnetic ground state phase diagram of the system. 
The outline of the paper is as follows. In the next section the classical cluster method will be outlined and phases will be obtained. In section III we present our numerical results of the exact diagonalization calculations on the ground state properties of the model. In section IV, the entanglement between different spins will be investigated by calculating the concurrence function. Finally we conclude and summarize our results in section V.

\section{CLASSICAL PHASE DIAGRAM}

The Hamiltonian of the 1D spin- $1 / 2$ frustrated ferromagnetic model in presence of a uniform DM interaction is defined as

$$
H=\sum_{j=1}^{N}\left[J_{1} \vec{S}_{j} \cdot \vec{S}_{j+1}+J_{2} \vec{S}_{j} \cdot \vec{S}_{j+2}+\vec{D} \cdot\left(\vec{S}_{j} \times \vec{S}_{j+1}\right)\right],
$$

where $\vec{D}=D \hat{z}$. This Hamiltonian shows a model with fully broken spin rotational symmetry. It is useful to begin with simple classical considerations that shed light on the possible ground state of the fully quantum mechanical problem. When $S$ is large, it is adequate to substitute $\vec{S}_{j}=S\left(\sin \theta_{j} \cos \phi_{j}, \sin \theta_{j} \sin \phi_{j}, \cos \theta_{j}\right)$ in Eq. (2) and find solutions that satisfy $\partial H / \partial \theta_{j}=0$ and $\partial H / \partial \phi_{j}=0$ for all $j$. But, here we use the rather unknown cluster method ${ }^{30,31}$ of Lyons and Kaplan (LK method). Briefly recall that method with assuming periodic boundary condition. Then we can easily see that Eq. (2) can be rewritten as:

$$
H_{c}=\sum_{j} h_{c}\left(\vec{S}_{j-1}, \vec{S}_{j}, \vec{S}_{j+1}\right),
$$

where the "cluster energy" involve three neighboring spins is

$$
\begin{aligned}
h_{c}\left(\vec{S}_{1}, \vec{S}_{2}, \vec{S}_{3}\right) & =\frac{1}{2}\left\{J_{1}\left(\vec{S}_{1} \cdot \vec{S}_{2}+\vec{S}_{2} \cdot \vec{S}_{3}\right)\right. \\
& \left.+\vec{D} \cdot\left(\vec{S}_{1} \times \vec{S}_{2}+\vec{S}_{2} \times \vec{S}_{3}\right)\right\} \\
& +J_{2} \vec{S}_{1} \cdot \vec{S}_{3} .
\end{aligned}
$$

It is clear that

$$
H_{c} \geq \sum_{j} \min h_{c}\left(\vec{S}_{j-1}, \vec{S}_{j}, \vec{S}_{j+1}\right),
$$

so easily one can find the minimum of $h_{c}$. If the corresponding state propagates, i.e. if there is a state of the whole system such that every set of three successive spins gives the minimum $h_{c}$, then according to Eq. (5), this state will be a ground state of the Hamiltonian. This is the main idea of the LK cluster method as applied to the present problem. This method has also been applied

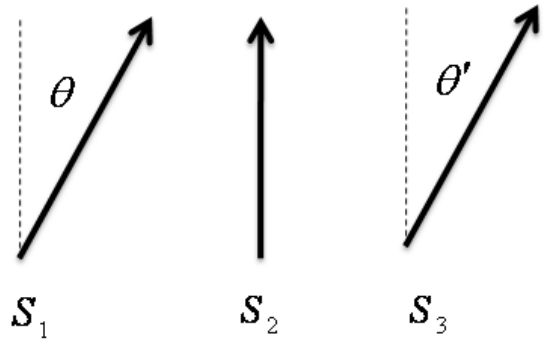

FIG. 1: (color online). The schematic picture of angles made by the spins in a cluster of three spins.

for models with higher dimensions or with open boundary conditions ${ }^{30}$.

Now let's minimize $h_{c}$. Because the DM vector is selected along the $z$ axis, we consider only coplanar (spin dimensionality $\mathrm{d}=2$, i.e. $\mathrm{XY}$ spins) state. Without loss of generality label the angles made by the end spins with the central spin $\theta, \theta^{\prime}$ (Fig 1). So the cluster energy is became

$$
\begin{aligned}
h_{c}\left(\theta, \theta^{\prime}\right) & =\frac{\left|J_{1}\right|}{8}\left\{-\cos \theta-\cos \theta^{\prime}+\gamma\left(\sin \theta-\sin \theta^{\prime}\right)\right. \\
& \left.+2 \alpha \cos \left(\theta-\theta^{\prime}\right)\right\},
\end{aligned}
$$

where $\gamma=\frac{D}{\mid J_{1}}$. Differentiating gives the conditions for stationarity

$$
\begin{gathered}
\frac{\left|J_{1}\right|}{8}\left\{\sin \theta+\gamma \cos \theta-2 \alpha \sin \left(\theta-\theta^{\prime}\right)\right\}=0 \\
\frac{\left|J_{1}\right|}{8}\left\{\sin \theta^{\prime}-\gamma \cos \theta^{\prime}+2 \alpha \sin \left(\theta-\theta^{\prime}\right)\right\}=0 .
\end{gathered}
$$

Before going through the Eq. (6), we check two simplified cases as follow.

First case: We have put $D=0$ and the frustrated ferromagnetic Heisenberg model recovered which well studied numerically and analytically. In this case, solutions are:

$$
\begin{aligned}
\left(\theta, \theta^{\prime}\right) & =(0,0),(\pi, \pi)(0, \pi)(\pi, 0) \quad(\text { Ising }- \text { type }) \\
\left(\theta, \theta^{\prime}\right) & =\left(\theta_{0},-\theta_{0}\right) \quad(\text { chiral }- \text { type }), \quad \text { where } \\
\cos \theta_{0} & =\frac{1}{4 \alpha} .
\end{aligned}
$$

The $(\pi, \pi)$ solution, which leads to the ordinary antiferromagnetic state, is never lowest because we have assumed $J_{1}<0$. The $(0,0)$ solution obviously propagates as the ferromagnetic state. The solutions $(\pi, 0),(0, \pi)$,i.e. $(\downarrow, \uparrow, \uparrow),(\uparrow, \uparrow, \downarrow)$ plus their degenerate reversed spin counterparts can easily be seen to propagate in the up-updown-down state ${ }^{30}$. 


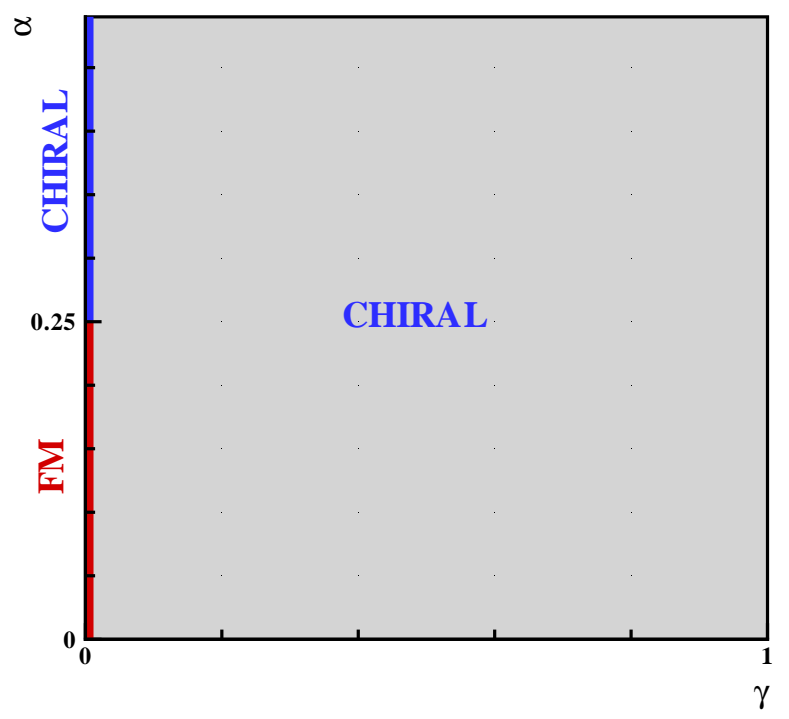

FIG. 2: (color online). The classical ground state magnetic phase diagram.

We listed the energies for the various stationary solutions:

$$
\begin{aligned}
h_{\text {ferro }} & =h_{c}(0,0)=\frac{\left|J_{1}\right|}{8}(-2+2 \alpha), \\
h_{\text {uudd }} & =h_{c}(0, \pi)=\frac{\left|J_{1}\right|}{8}(-2 \alpha), \\
h_{\text {chiral }} & =h_{c}\left(\theta_{0},-\theta_{0}\right)=\frac{\left|J_{1}\right|}{8}\left(-\frac{1}{4 \alpha}-2 \alpha\right) .
\end{aligned}
$$

By equating these energies in pairs we found only one critical point, $\alpha_{c}=0.25$. The ground state is in the ferromagnetic phase for $\alpha<0.25$ and is in the chiral phase for $\alpha>0.25$.

Second case: We have put $J_{2}=0$ (or $\alpha=0$ ) which means we have just 1D isotropic ferromagnetic Heisenberg model plus a uniform DM interaction. In this case there is only one solution:

$$
\begin{aligned}
\left(\theta, \theta^{\prime}\right) & =\left(\theta_{0},-\theta_{0}\right) \quad \text { (chiral type) where } \\
\tan \theta_{0} & =-\gamma .
\end{aligned}
$$

Which means that immediately after turning on the DM interaction, the long range chiral order is created in the classical ground state phase diagram. In principle, as soon as the DM interaction is applied, the ground state of the system undergoes a phase transition from the ferromagnetic phase into a chiral phase.

It is better to emphasis that the induced effects of quantum fluctuations on the 1D antiferromagnetic Heisenberg model with DM interaction had been studied theoretically and experimentally ${ }^{32}-34$. From quantum point of view, it has been found that the DM interaction induces the chiral phase which remains stable even in presence of a uniform magnetic field ${ }^{32}$.

Third case: Now we consider the 1D frustrated ferromagnetic model with DM interaction. The solutions for general equations (6) are

$$
\begin{aligned}
\left(\theta, \theta^{\prime}\right) & =(0,0) \quad \text { where } \gamma=0 \\
\left(\theta, \theta^{\prime}\right) & =(0, \pi)=(\pi, 0) \quad \text { where } \gamma=0 \\
\left(\theta, \theta^{\prime}\right) & =\left(\theta_{0},-\theta_{0}\right) \quad \text { where } \\
4 \alpha & =\frac{1}{\cos \theta_{0}}+\frac{\gamma}{\sin \theta_{0}} .
\end{aligned}
$$

These results show, the ferromagnetic and up-up-downdown orders exist only in the absence of the DM interaction. As soon as the DM interaction increases from zero, the ground state of the system goes to the chiral phase, independent of the frustrated parameter $\alpha$ (Fig 2). Thus, using the cluster LK method, the classical ground state phase diagram of the $1 \mathrm{D}$ spin- $1 / 2$ frustrated ferromagnetic model with added uniform DM interaction consists of a single phase: "chiral".

\section{QUANTUM PHASE DIAGRAM}

In this section, to explore the nature of the spectrum and the quantum phase transition, we used the Lanczos method to diagonalize numerically chains with length up to $N=24$.

First, in different subspaces we have computed the ground state energy of chains with $J_{1}=-1$ and different values of the DM interaction as a function of the parameter $\alpha$. The setting of boundary conditions and observation of energy with respect to change of parameters is important for precise analysis of wave state ${ }^{39}$, but because of our limitation and size effects, we have just set the periodic boundary condition. In Fig. 3. we present results of these calculations for the value of DM vector $D=0.05$ and chain size $N=20$. It can be seen that the ground state energy for weak DM interaction is nearly degenerate up to the first critical frustration $\alpha_{c_{1}}=0.24 \pm 0.01$, which value is obtained by extrapolation technique. Also degeneracy in the mentioned region shows that in the absence of the frustration, $\alpha=0$, the spectrum of the model is gapless. By turning on the frustration, spectrum remains gapless up to the first critical value of the frustration, $\alpha_{c_{1}}=0.24 \pm 0.01$. As soon as the frustration increases from $\alpha_{c_{1}}$, the ground state is nondegenerate and exists in the subspace with total $S^{z}=0$. Fig. 3 is also good analysis, especially for the proof of ferromagnetic sate.

From the viewpoint of the symmetry our model is completely different than the models studied in the literature $36-38,40-46$. The latter models have the $Z_{2} \times$ $U(1)$ symmetry. In this case, the absence of the chiral LRO is natural, and the spontaneous breaking of the $Z_{2}$ 


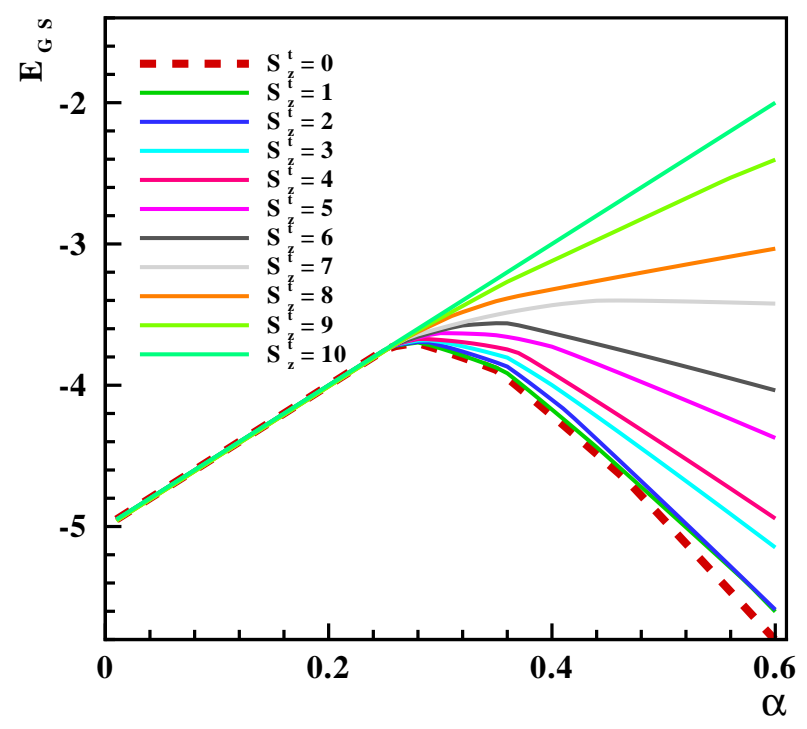

FIG. 3: (color online). Ground state energy of the system in different subspaces versus the frustration parameter $\alpha$ for chain with length $N=20$ and DM vector $D=0.05$.

chiral symmetry, which resulted in the chiral LRO, is interesting and was indeed the main topic in those studies. On the other hand, in the present model, the $Z_{2}$ chiral symmetry is broken by the DM interaction explicitly. Therefore, the appearance of the chiral LRO should be natural; if the LRO is absent, it should be regarded as a surprising result and there must be a very interesting mechanism which recovers the symmetry behind the result.

In order to recognize the different quantum phases, we have implemented the Lanczos algorithm on finite size chains to calculate the lowest eigenstate. The first insight into the nature of different phases can be obtained studying the chiral order parameter. It has been shown that the DM interaction can create the chiral phase in the ground state phase diagram of the spin systems ${ }^{32.33}$, which is characterized by nonzero value of the chirality

$$
\chi^{z}=\frac{1}{N} \sum_{j}\left\langle\chi_{j}^{z}\right\rangle
$$

where $\chi_{j}^{z}=\left(\mathbf{S}_{j} \times \mathbf{S}_{j+1}\right)^{z}$. One should note that there are two different types of the chiral ordered phases, gapped and gapless $\frac{45.47}{4}$. In Fig. 4(a), we have presented calculated results on the chiral order parameter as a function of the frustration parameter $\alpha$ for a value of DM vector $D=0.05$, including different chain lengths $N=16,20,24$. As is clearly seen from this figure, there is no long-range chiral order along the $z$ axis in the regions $\alpha<\alpha_{c_{1}}$ and $\alpha>\alpha_{c_{2}}$. However, in the intermediate region, $\alpha_{c_{1}}<\alpha<\alpha_{c_{2}}$, the ground state shows a profound chiral order in the $z$ direction. Since the study
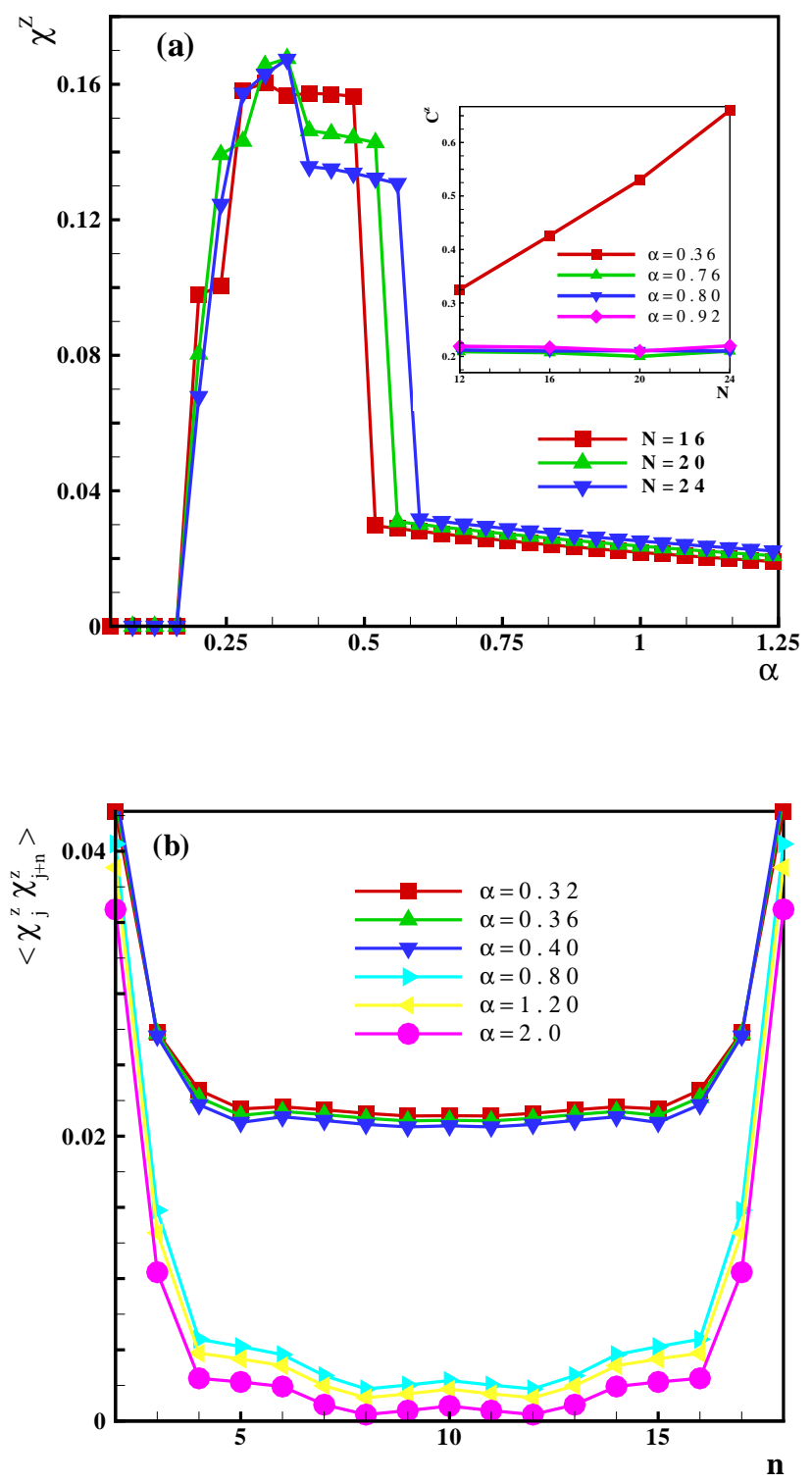

FIG. 4: (color online). (a) The chiral order parameter $\chi^{z}$ as a function of the frustration parameter $\alpha$ for the value of DM vector $D=0.05$, including different chain lengths $N=$ $16,20,24$. In the inset the chiral correlation function is plotted as a function of the chain length $N$ for the value of frustration $\alpha=0.36$. The diverging behavior of the correlation function shows that the chiral ordering in the intermediate region is true long range. (b) The correlation $\left\langle\chi_{j}^{z} \chi_{j+n}^{z}\right\rangle$ as a function of $n$ for chain length $N=20$ and $D=0.05$.

of correlation functions can give us deep insight into the characteristics of the ground state, we define the chiral correlation function as

$$
C^{z}=\sum_{n=1}^{N}\left\langle\chi_{j}^{z} \chi_{j+n}^{z}\right\rangle .
$$


It is important that in a phase with true long-range order the correlation functions should diverge as $N \longrightarrow \infty$. To check the existence of the chiral long-range order in the thermodynamic limit $N \longrightarrow \infty$ of the system, we have plotted in the inset of Fig. 4(a) the $N$ dependence of $C^{z}$ for different values of frustration in the regions $\alpha>\alpha_{c_{1}}$. As is seen from this figure, only in the intermediate region $\alpha_{c_{1}}<\alpha<\alpha_{c_{2}}$, there is a diverging behavior which shows that the chiral order in the intermediate region is true long-range order. On the other hand, the constant value in the region $\alpha>\alpha_{c_{2}}$ shows that the $C^{z} / N$ takes zero value in the thermodynamic limit $N \longrightarrow \infty$. For grater emphasis, we have plotted in Fig. 廿(b), the correlation function $\left\langle\chi_{j}^{z} \chi_{j+n}^{z}\right\rangle$ as a function of $n$ for a chain length $N=20$. Clearly is seen that the long distance correlation of the chirality only exist in the region $\alpha_{c_{1}}<\alpha<\alpha_{c_{2}}$. The mirror symmetry is the result of the periodic boundary condition. Therefore, by studying the ground state energy and the chiral order parameter, we showed that in the intermediate region $\alpha_{c_{1}}<\alpha<\alpha_{c_{2}}$, the $1 \mathrm{D}$ spin- $1 / 2$ frustrated ferromagnetic model with added DM interaction is in the gapped chiral order phase.

In what follows we will present our numerical study about the presence of dimer phase. In order to understand the nature of the dimer phase let us assume we have a $1 \mathrm{D}$ frustrated antiferromagnetic model, where the sign of $J_{1}$ will be changed through the $\pi$ rotations around the $z$ axis of the spins on every second sites and the model will be transformed to the 1D frustrated ferromagnetic model. From the fact that the ground state at the Majumdar-Ghosh point $\left(J_{2}=J_{1} / 2\right)$ is given by the product of singlet dimmers $\left(\frac{1}{\sqrt{ } 2}(|\uparrow \downarrow\rangle-|\downarrow \uparrow\rangle)\right)^{6}$. Through the above $\pi$-rotation transformation, the dimer unit for $J_{2}=-J_{1} / 2$ is replaced by the triplet state $\frac{1}{\sqrt{ } 2}(|\uparrow \downarrow\rangle+|\downarrow \uparrow\rangle) \frac{12}{2}$. To this reason, to find additional insight into the different phases we are focused on existing of the dimer phase in our 1D frustrated ferromagnetic model. The order parameter characterizing the dimer phase is given as ${ }^{?}$

$$
d=\frac{1}{N} \sum_{i}(-1)^{i}\left\langle S_{i} \cdot S_{i+1}\right\rangle
$$

This order parameter shows alternating signs along the spin chain. Our results for the DM vector value $D=0.05$ and different chain sizes $N=16,20,24$ are presented in Fig. 5. As can clearly be seen from this figure, in the Luttinger liquid region, $\alpha<\alpha_{c_{1}}$, there is not any longrange dimer order. As soon as the frustration increases from the first critical value, the dimer ordering increases from zero very rapidly. The oscillations of the dimer order in the intermediate region at finite $N$ are the result of level crossing between ground state and excited states of the model. On the other hand, overlapping of the numerical results in the region $\alpha>\alpha_{c_{2}}$, shows a divergent behavior of the correlation function of the dimer order parameter by increasing the size of chain $N$. This justifies

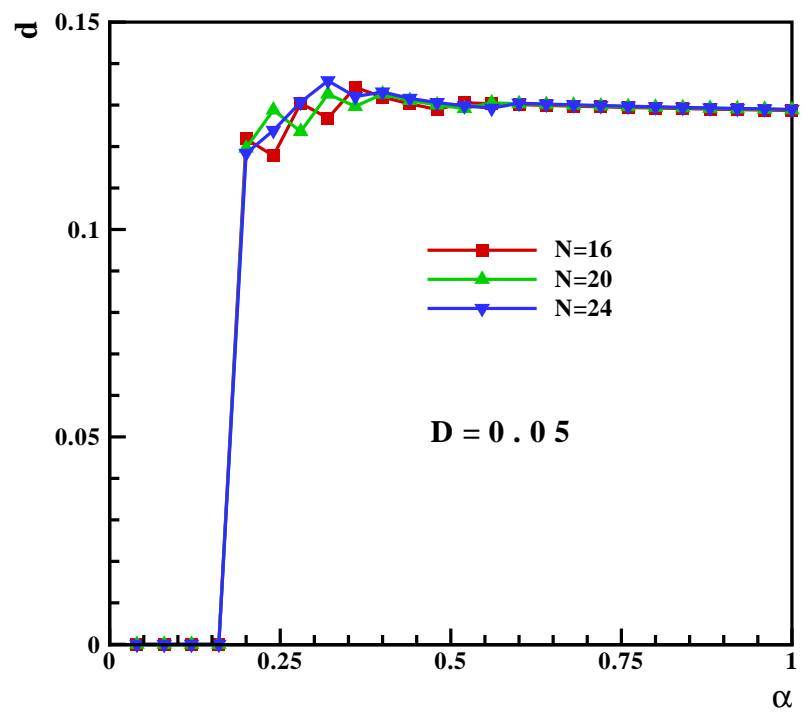

FIG. 5: (color online). The dimer order parameter, $d$, as a function of the frustration parameter $\alpha$ for the value of DM vector $D=0.05$, including different chain lengths $N=$ $16,20,24$.

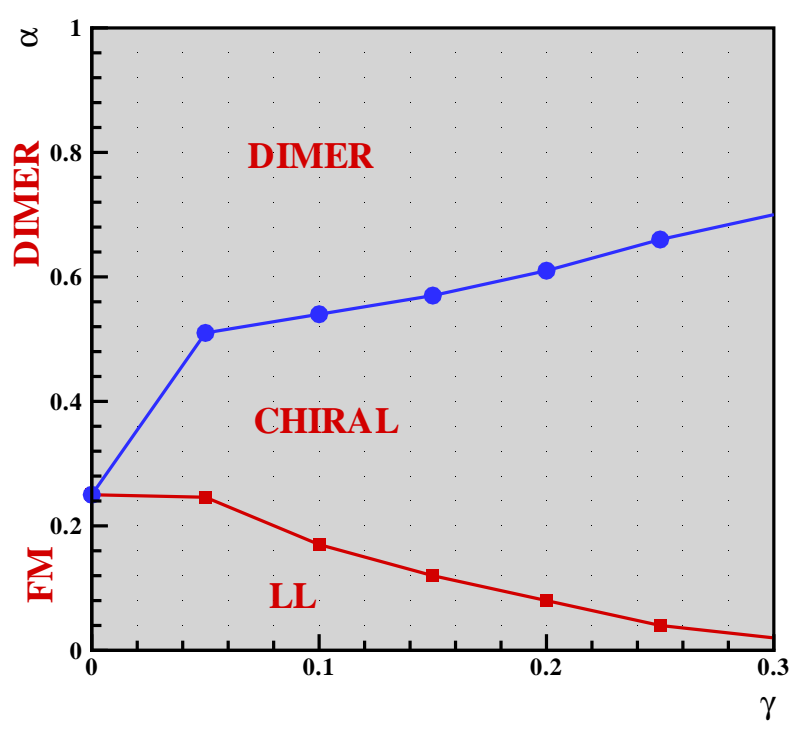

FIG. 6: (color online). The quantum ground state magnetic phase diagram.

that the true long-range dimer order exists in the region $\alpha>\alpha_{c_{2}}$ of the ground state phase diagram.

We have to mention that for other values of the DM interaction $(D \leq 0.3)$, we did the same numerical experiment and found the same quantum picture of the ground state magnetic phase diagram contains of: (I.) gapless Luttinger liquid phase in the region $\alpha<\alpha_{c_{1}}$ (II.) gapped 
chiral phase in the intermediate region $\alpha_{c_{1}}<\alpha<\alpha_{c_{2}}$ and (III.) gapped dimer phase for $\alpha>\alpha_{c_{2}}$ (Fig 6).

\section{CONCURRENCE}

In this section we study the entanglement of formation as a measure of the entanglement. Entanglement stands some similarity to classical correlation, but it differs in some important respects, including the fact that entangled objects can violate Bells inequality $\underline{48}$. May be one of the most characteristic differences is this: if two similar quantum objects are completely entangled with each other, then neither of them can be at all entangled with any other object, but there is no such restriction on classical correlations 49 .

We compute the entanglement between two sites which is known as the concurrence ${ }^{50.52}$

$$
C_{l m}=2 \max \left\{0, C_{l m}^{(1)}, C_{l m}^{(2)}\right\},
$$

where

$$
\begin{aligned}
C_{l m}^{(1)} & =\sqrt{\left(g_{l m}^{x x}-g_{l m}^{y y}\right)^{2}+\left(g_{l m}^{x y}+g_{l m}^{y x}\right)^{2}} \\
& -\sqrt{\left(\frac{1}{4}-g_{l m}^{z z}\right)^{2}-\left(\frac{M_{l}^{z}-M_{m}^{z}}{2}\right)^{2}} \\
C_{l m}^{(2)} & =\sqrt{\left(g_{l m}^{x x}+g_{l m}^{y y}\right)^{2}+\left(g_{l m}^{x y}-g_{l m}^{y x}\right)^{2}} \\
& -\sqrt{\left(\frac{1}{4}+g_{l m}^{z z}\right)^{2}-\left(\frac{M_{l}^{z}+M_{m}^{z}}{2}\right)^{2}},
\end{aligned}
$$

where $M^{z}$ is magnetization along $z$ axis and $g_{l m}^{\mu \nu}=$ $\left\langle S_{l}^{\mu} S_{m}^{\nu}\right\rangle$ is the correlation function between spins on sites $l$ and $m$.

The numerical Lanczos results on the concurrence are shown in Fig. 7 From our numerical results we found that the concurrence of two spins that are nearest neighbor is equal to zero in all regions of the quantum ground state phase diagram. In this figure the concurrence of one spin and its next nearest neighbor is plotted as a function of the frustration parameter $\alpha$ for chain lengths $N=16,20,24$ and DM vector $D=0.05$. In the Luttinger liquid and dimer phases, the correlation between different components, $g_{l m}^{x y}, g_{l m}^{y x}$ is equal to zero and only in the chiral phase has non-zero value. As it can be seen from Fig. [7, in the Luttinger liquid region, $\alpha<\alpha_{c_{1}}$, since the correlations $g_{l m}^{x x}, g_{l m}^{y y}$ are equal, therefore the concurrence is equal to zero. On the other hand in the intermediate chiral region, $\alpha_{c_{1}}<\alpha<\alpha_{c_{2}}$, due to non-zero value of the correlations between different components the concurrence increases by increasing the frustration and at the second critical value $\alpha=\alpha_{c_{2}}$ is maximum. In sector of the dimer ground state, $\alpha>\alpha_{c_{2}}$, the correlations $g_{l m}^{x x}$, $g_{l m}^{y y}$ are equal and close to the maximum value $\frac{121}{4}$. In this case, as it is clearly seen from the Fig. 17, the concurrence has the almost constant value that obeys from the equation $C_{l m}=\left|g_{l m}^{x x}+g_{l m}^{y y}\right| \simeq 0.5$. The deviation from the

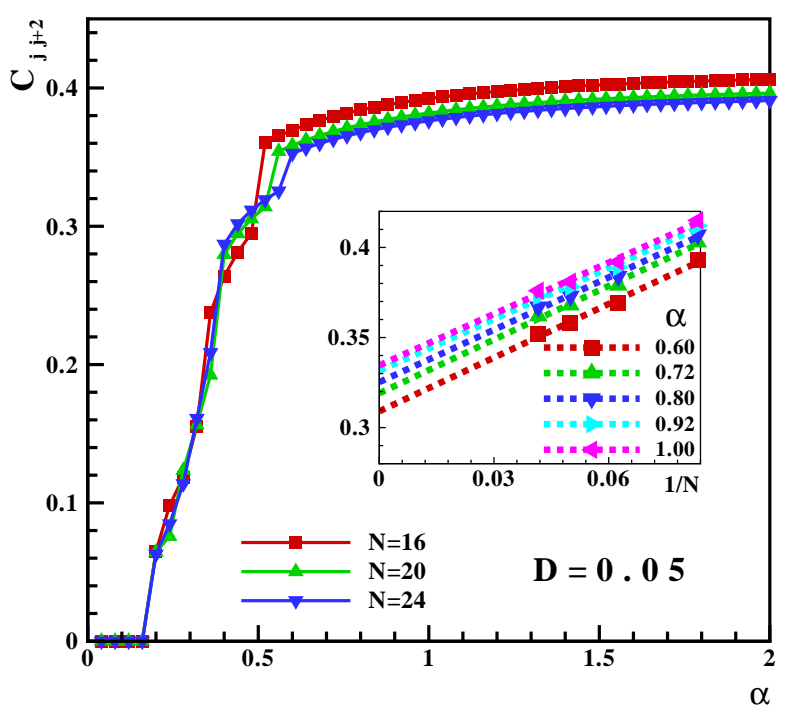

FIG. 7: (color online). The entanglement between next nearest neighbors $C_{j}{ }_{j+2}$ as a function of the frustration parameter $\alpha$ for the value of DM vector $D=0.05$, including different chain lengths $N=16,20,24$. The inset shows the concurrence as a function of the inverse length $1 / N$ for different values of the frustration $\alpha=0.60,0.72,0.80,0.92,1.00$. Finite linear extrapolated results show that in the thermodynamic limit $N \longrightarrow \infty$, the next nearest neighbors are entangled in the dimer phase.

saturation value 0.5 is a result of the induced quantum fluctuations by DM interaction. In the inset of Fig. (7) the $N$-dependence of the concurrence is investigated for different values of the frustration in the dimer sector of the ground state phase diagram. Finite linear extrapolated results show that in the thermodynamic limit $N \longrightarrow \infty$, the next nearest neighbors are really entangled in the dimer phase. Thus, in the 1D frustrated ferromagnetic spin- $1 / 2$ model in presence of the DM interaction, only the next nearest neighbors are entangled in the gapped chiral and dimer phases.

It is expected that quantum nature of entanglement might provide explanatory and predictive power for the investigation of quantum phase transitions ${ }^{51,52}$. Thus concurrence -pairwise entanglement- which refers to quantum correlations has emerged as one of the important tools. In our model Eq.(2), only the next nearest neighbors quantum correlation lead to concurrence have different nonzero value in the gapped chiral and dimer phases and zero value in the Luttinger liquid phase.

On the other hand, entanglement generation and distribution are problems of central importance in performing quantum-information(QI) tasks, like teleportation ${ }^{53}$ and quantum cryptography $\underline{54}$. From the QI perspective, it would be attracting to create sizable entanglement between particles that are located at a distance larger than 


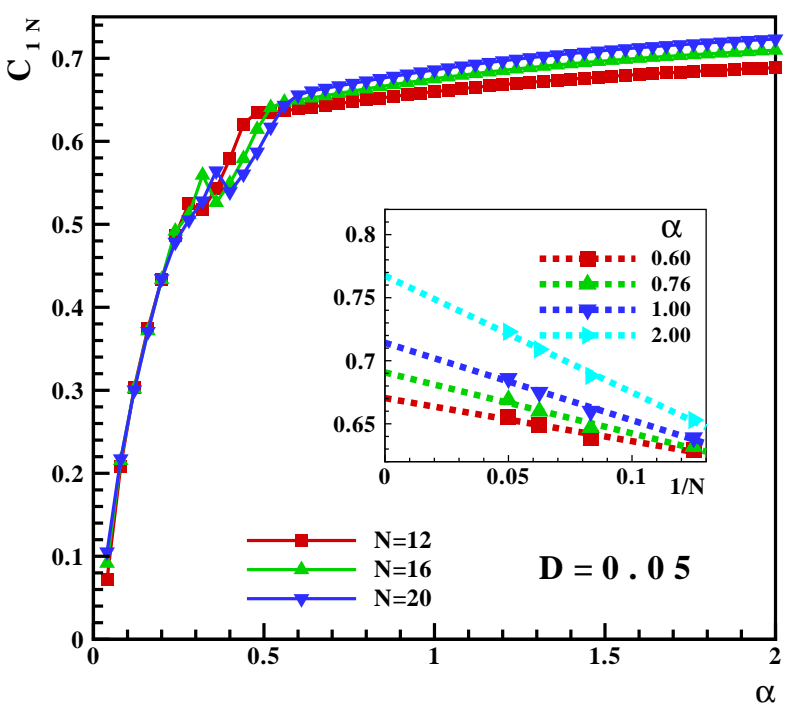

FIG. 8: (color online). The entanglement between end spins as a function of $\alpha$, with $D=0.05$ for different chain lengths up to $N=20$, with open boundary condition. The inset shows $C_{1 N}$ as a function of the inverse length $1 / N$ for different values of the frustration $\alpha=0.60,0.76,1.00,2.00$. The extrapolated results in the thermodynamic limit $N \longrightarrow \infty$ are less than one.

a few sites. This fact naturally leads to the concepts of long distance entanglement (LDE) as a sort of quantum order parameter 55 . There are some models same as the dimerized-frustrated model, spin-1 Heisenberg chain with biquadratic interaction and so on, which able to produce $\mathrm{LDE}^{55}$. Here we are interested to check this feature in our model. As we showed in previous sections our spin frustration model leads to dimerized phase if the parameter $\alpha$ exceeds a certain critical value $\left(\alpha_{c_{2}}\right)$, so the process of dimerization with the open boundary condition enables frustrated model to produce LDE. In Fig. 8, we give the numerical Lanczos results on the long distance entanglement of frustrated Heisenberg chain in the presence of the DM interaction, particularly $D=0.05$. As it can be seen from Fig. 8, in contrast next-nearest neighbor entanglement, the system has got nonzero LDE which increases rapidly by increasing $\alpha$ up to $\alpha_{c_{2}}$ and then after that it reaches its saturation value about $C_{1-N} \simeq 0.7$. In the inset of Fig. 8 the $N$-dependence of the LDE is investigated for different values of the frustration in the dimer sector of the ground state phase diagram. Linear extrapolated results show that in the thermodynamic limit $N \longrightarrow \infty$, the end-spins are entangled in the dimer phase for open boundary conditions.

\section{CONCLUSION}

To summarize, we studied the effect of a uniform Dzyaloshinskii-Moriya (DM) interaction on the ground state phase diagram of the one-dimensional (1D) isotropic frustrated ferromagnetic spin-1/2 model using the analytical cluster method and numerical Lanczos technique. Our classical analysis results based on LK method show that immediately after turning on the DM interaction, the ground state of the system goes to the chiral phase, independent of the frustration parameter $\alpha=\frac{J_{2}}{\left|J_{1}\right|}$. Thus, we concluded that the classical ground state phase diagram of the 1D frustrated ferromagnetic spin-1/2 model with added uniform DM interaction consists of a single phase: "chiral".

To find the quantum corrections of adding the DM interaction, we did a very accurate numerical experiment. We implemented the Lanczos algorithm to find the ground state in finite chains. Based on the numerical results of the order parameters and correlation functions, we identified three different phases. In the region $\alpha<\alpha_{c_{1}}$, the ground state of the system is in the Luttinger liquid phase. In the region $\alpha>\alpha_{c_{2}}$ the true longrange dimer order exists in the ground state phase diagram and in the intermediate region $\alpha_{c_{1}}<\alpha<\alpha_{c_{2}}$, the system finds in the gapped chiral phase.

On the other hand, we tried to answer this question: "Are the spins entangled in different sectors of the ground state phase diagram?" Our numerical results for the entanglement showed that the nearest neighbor spins are not entangled in all regions of the quantum ground state phase diagram. But, the next nearest neighbor spins are entangled in the gapped chiral and dimer phases, and became saturate in the region $\alpha>\alpha_{c_{2}}$, which can be take it as another witness the presence of dimer phase. We have even checked this model with open boundary conditions as an candidate for LDE model, where calculations showed that the model reaches its saturated value around $\alpha_{c_{2}}$ which is more than next nearest neighbor entanglement case $C_{1 N}>C_{j} j+2$.

\section{ACKNOWLEDGMENTS}

It is our pleasure to thank T. Kaplan, G. I. Japaridze, T. Vekua, A. Akbari, J. Abouie, S. H. Sadat, M. R. Soltani, G. Rigolin, T. Nishino, and T. Hikihara for very useful comments and useful suggestions. We are also grateful to S. N. Rasouli for reading our manuscript.
1 Highly Frustrated Magnetism 2008, J. of Phys.: Conference Series 145 (2009); Proceedings of the Highly Frus- trated Magnetism 2003 Conference, J. Phys. Cond. Mat. 16, n. 11 (2004). 
${ }^{2}$ F. D. Haldane, Phys.Rev. B 25, R4925 (1982).

3 T. Tonegawa and I. Harada, J. Phys. Soc. Jpn. 56, 2153 (1987).

4 K. Nomura and K. Okamoto, Phys. Lett. A 169, 433 (1992).

${ }^{5}$ R. Bursill, G. A. Gehring, D. J. J. Farnell, J. B. Parkinson, T. Xiang and C. Zeng, J. Phys.: Condens. Matter 7, 8605 (1995).

${ }^{6}$ C. K. Majumdar and D. K. Ghosh, J. Math. Phys. 10, 1388 (1969).

7 Steven R. White, Ian Affleck, Phys. Rev. B 54, 9863 (1996).

8 G. Bouzerar, A. P. Kampf, and G. I. JAparidze, Phys. Rev. B 58, 3177 (1998).

9 Manoraja Kumar, Z. G. Soos, Diptiman Sen, and S. Ramasesha, Phys. Rev. B 81, 104406 (2010).

10 Manoraja Kumar, S. Ramasesha, Z. G. Soos, Phys. Rev. B 81, 054413 (2010).

11 T. Tonegawa and I. Harada, J. Phys. Soc. Jpn. 58, 2902 (1989).

12 A. V. Chubukov, Phys. Rev. B 44, R4693 (1991).

13 D. C. Cabra, A. Honecker and P. Pujol, Eur. Phys. J. B 13, 55 (2000).

14 V. Ya. Krivnov and A. A. Ovchinnikov, Phys. Rev. B 53, 6435 (1996).

15 T. Hamada, J. Kane, S. Nakagawa and Y. Natsume, J. Phys. Soc. Jpn. 57, 1891 (1988).

16 D. V. Dmitriev , V. Ya. Krivnov and A. A. Ovchinnikov, Phys. Rev. B 56, 5985 (1997).

17 C. Itoi and S. Qin, Phys. Rev. B 63, 224423 (2001).

18 D. Allen and D. Senechal, Phys. Rev. B 55, 299 (1997).

19 A. A. Nersesyan, A. Q. Gogolin and F. H. L. Essler, Phys. Rev. Lett. 81, 910 (1998)

20 D. V. Dmitriev, V. Ya. Krivnov, and J. Richter, Phys. Rev. B 75, 014424 (2007).

21 S. Mahdavifar, J. Phys.: Condens. Matter 20, 335230 (2008).

22 Y. Mizuno, T. Tohyama, S. Maekawa, T. Osafune, N. Motoyama, H. Eisaki and S. Uchida, Phys. Rev. B 57, 5326 (1998).

23 M. Hase, H. Kuroe, K. Ozawa, O. Suzuki, H. Kitazawa, G. Kido and T. Sekin, Phys. Rev. B 70, 104426 (2004).

24 S. F. Solodovnikov and Z. A. Solodovinkova, J. Struct. Chem. 38, 765 (1997).

25 I. Dzyaloshinskii, J. Phys. Chem. Solids 4, 241 (1958).

26 T. Moriya, Phys. Rev 120, 91 (1960).

27 M. Enderle, C. Mukherjee, B. Fak, R. K. Kremer, J.-M. Broto, H. Rosner, S.-L. Drechsler, J. Richter, J. Malek, A. Prokofiev, W. Assmus, S. Pujol, J.-L. Raggazzoni, H. Rakoto, M. Rheinstadter and H. M. Ronnow: Europhys. Lett. 70 (2005) 237.

${ }^{28}$ Y. Naito, K. Sato, Y. Yasui, Y. Kobayashi, Y. Kobayashi and M. Sato, J. Phys. Soc. Jpn. 76023708 (2007).

29 S. Furukawa, M. Sato, S. Onoda, Phys. Rev. Lett. 105, 257205 (2010)

30 D. H. Lyons and T. A. Kaplan, J. Phys. Chem. Solids 25, 645 (1964).

31 T. A. Kaplan, Phys. Rev. B 80, 012407 (2009).

32 S. Mahdavifar, M. R. Soltani, A. A. Masoudi, Eur. Phys. J. B 62, 215 (2008).

33 M. R. Soltani, S. Mahdavifar, A. Akbari, J. Supercond. Nov. Magn. 23, 1369 (2010).

34 I. Garate, I. Affleck, Phys. Rev. B 81, 144419 (2010).

35 R. Jafari, A. Langari, Phys. Rev. B 76, 014412 (2007).

36 D. V. Dmitriev, V. Ya. Krivnov, Phys. Rev. B 77, 024401 (2008); D. V. Dmitriev, V. Ya. Krivnov, Phys. Rev. B 79, 054421 (2009); D. V. Dmitriev, V. Ya. Krivnov, Phys. Rev. B 81, 054408 (2010).

37 F. Heidrich-Misner, I. P. McCulloch, A. K. Kolezhuk, Phys. Rev. B 80, 144417 (2009).

38 Shunsuke Furukawa, Mashahiri Sato, Akira Furusaki, Phys. Rev. B 81, 094430 (2010).

39 A. Gendiar and T. Nishino Phys. Rev. B 71, 024404 (2005).

40 F. Heidrich-Misner, A. Honecker, T. Vekua, Phys. Rev. B 74, 020403(R) (2006).

41 T. Vekua, A. Honecker, H-J. Mikeska, F. Heidrich-Misner, Phys. Rev. B 76, 174420 (2007).

42 Lars Kecke, Tsutomu Momoi, Akira Furusaki, Phys. Rev. B 76, 060407 (2007).

43 Toshiya Hikihara, Lars Kecke, Tsutomu Momoi, Akira Furusaki, Phys. Rev. B 78, 144404 (2008).

44 J. Sudan, A. Luscher, A. Laeuchli, Phys. Rev. B 80, 140402(R) (2009).

45 T. Hikihara et al., J. Phys. Soc. Jpn 69, 259 (2000).

46 T. Hikihara, M. Kaburagi, and H. Kawamura, Phys. Rev. B 63, 174430 (2001).

47 M. Kaburagi, H. Kawamura, T. Hikihara, J. Phys. Soc. Jpn 68, 3185 (1999).

48 J. S. Bell, Physics (Long Island City, N.Y.) 1, 195 (1964).

49 K. M. OConnor and W. K. Wootters Phys. Rev. A . 63, 052302 (2001).

${ }^{50}$ W. K. Wooters, Phys. Rev. Lett. 80, 2245 (1998).

51 Osterloh, R., Amico, L., Falci, G., Fazio, R.: Nature (London) 416, 608 (2002).

52 L. Amico, R. Fazio, A. Osterloh, and V. Vedal, Rev. Mod. Pys. 80, 517 (2008).

53 D. Boschi, S. Branca, F. De Martin, L. Hardy, and S. Popescu, Phys. Rev. Lett, 80, 1121 (1998).

54 N. Gisini, G. Ribordy, W. Tittel, and H. Zbindeh, Rev. Mod. phys.74, 145 (2002)

55 L. Campos Venuti, C. Degli Espositi Boschi, and M. Roncaglia, Phys. Rev. Lett. 96, 247206 (2006). 